jetzt nicht künstlich dargestellt werden konnte. Diese Base wurde mit dem Namen Propylamin bezeichnet und ihre Zusammensezzung durch die Formel $\left.\mathrm{C}_{6} \mathrm{H}_{4} \mathrm{~N}={ }_{\mathrm{C}_{6}} \mathrm{H}_{7}\right\} \mathrm{N}$ ausgedrückt. Wertheim hat num in der letzten Zeit eine vortheilhafte Darstellungsmethode dieser Substanz entdeckt. In grosser Menge erhält man sie bei der Destillation der Ileringslake mit Kali. Das Product der Destillation wird mit Chlorwasserstoflš̈ure gesăttigt, zur Trockue verdampft und der Rückstand mit Alkohol behandelt. Es lüst sich chlorwasserstoflsaures Propylamin auf, während als Ruickstand Salmiak bleibt. Im reinen Zustande ist dieses Salz in Wasser und Alkohol leicht löslich und sehr zerfliesslich.

Bei seinen fortgesetzten Untersuchungen über das Narcotin entdeckte Wertheim zwei Basen, dic sich zu dem Narcotin Blyth's $\mathrm{C}_{46} \mathrm{H}_{25} \mathrm{NO}_{44}$ verhalten, wie das Metlyglamin und Propylamin zum Aethylamin. Diese Körper sind das Methylnarcotin und Propylnarcolin. Die erste dieser Basen $\mathrm{C}_{44} \mathrm{H}_{23} \mathrm{NO}_{14}$ gicbt beim Behandeln mit detzkali Methylamin, die zweite $\mathrm{C}_{48} \mathrm{H}_{27} \mathrm{NO}_{14}$ giebt unter denselben Umständen Propylamin. Wertheim glaubl, dass das Narcotin Blyth's unter der Einvirkung der Alkalien Aethylamin geben wird.

\title{
XXXVII.
}

\section{Untersuchungen über die Vegetation unter dem Wasser lebender Pfianzen.}

\author{
Von \\ Clö̈z und Gratiolet.")
}

(Autr. d. Chim. et de phys. $\boldsymbol{X X X I I . p . 4 1 \text { ) }}$

Bonnet stellte gegen die Mitte des vorigen Jahrhunderts zahlreiche Versuche über die Bestimmung der Blätter an; ex machte unter anderen die Beobaclıtung, dass die Blătter vou

*) Ein karzer Anszng dieser Arbeit ans dem Compt. rend. befindet sich schon in d. Journ. Lil. p. 275.

D. Redact. 
Vegetabilien, wenn sie in das Wasser gebracht werden, sich, besonders dem Sonnenlichte ausgesetzt, an ihrer unteren Seite mit Luftblasen bedecken. Diese Beobachtungen führten jedoch nur zu ungenauen Interpretationen über die Natur dieser interessanten Erscheinung; sie blieben unfruchtbar bis zu dem Augenblicke, in welchem Priestley die Entdeckung machte, dass die Pflanzen mit dem Vermoggen begabt seien, der durch die Respiration der Thiere oder durch die Verbrennung einer Kerze veränderten Luft ihre ursprüngliche Reinheit zurückzugehen. Diese Beobachtung, welche den Menschen eine der schönsten Harmonien in der Natur enthüllte, wurde mit Enthusiasmus aufgenommen. Diese grosse Entdeckung blieb jedoch bis zu cinem gewissen Grade Hypothese. Priestley war nämlich in den Resultaten seiner Versuche auf gewisse Widersprüche gerathen, welche für ihn nicht zu beseiligen waren.

Ingenhouss, ein Bewunderer der Entdeckungen Priestley's unternahm es, das Paradoxon zu beseitigen; er stellte zahlreiche Versuche an, in welchen er gervissermaassen $B$ onnet mit Priestley zu vereinigen suchte. Auf diese Weise gelang es ihm nachzuweisen, dass die von Priestley entdeckte reinigende Eigenschaft nur den grünen Theilen der Pllanzen angehüre und nur unter dem Einflisse des Sonnenlichtes erfolge. Er nahm ausserdem an, dass die Pllanzen, wenn sie nicht unter diesen Bedingungen stehen, die Luft nicht nur nicht verbessern, sondern vielmelır verschlechtern.

Zur Vervollstindigung dieser schünen Untersuchungen erschien es nothwendig zu untersuchen, vermittelst welcher geheimnissvollen Processe diese Phinomene vor sich gehen; es blieb zu erforschen, ob die dem Sonnenlichte ausgesetzten Pflanzen aus der veränderten Luft elwas aufnehmen oder an dieselbe etwas abgeben. Senebier unternahm es, dieses Problem zu lösen; er wies durch zahlreiche Versuche nach, dass die grünen Pflanzentheile die durch die Verbrennung einer Kerze oder durch die Respiration der Thiere verunreinigte Luft dadurch verbesserten, dass sie aus derselben Kohlensăure aufnelımen und dafür Sauerstoff abgeben. Die Natur des Pbänomens war also erklärt. Noch blieb aber zu untersuchen übrig, in welcher Beziehung dieses Phānomen zil den Lebensfunctionen der Pflanze stünde. Th. v. Saussure versuchte zu bestimmen, welche Beziehung 
zwischen dem Volumen des absorbirten Kohlensăuregases und dem des abgegebenen Sauerstoffs stattindet; bei den Versuchen dieses Physikers war das Volumen des entwickelten Sauerstoffs stets kleiner als das der absorbirten Kohlensäure; der Antheil des in der Pflanze zurückgehaltenen Sauerstoffs war aber in dem ausgeathmeten Product durch ein fast gleiches Volumen Stickgas ersetzt.

Die Resultate der Versuche Sauss ure's veranlassten diesen Plysiker zu der Annahme, dass aller entwickelte Sauerstoff von der absorbirten Kollensăure herrülıre, dass aber ein Theil des Sauerstofls diese Säure von der Pflanze assimilirt werde. Sa ussure nahm ferner an, dass constant Stickstoff ausgehaucht werde und dass dieser Stickstoff von der Pflanzensubstanz berrühre.

Es geht aus dem Vorstehenden hervor, dass Saussure in der Geschichte dieser Erscheinung dem Wasser keine Betheiligung zuschrieb. Boussingault war der erste, welcher dieses Element in die Discussion der Frage einfülurte.

Den Ansichten dieses Chemikers zufolge kann der lurch die Ptlanze ausgegebene Sauerstoff durch die gemeinschaftiche Zersetzung der Kohlensaure und des Wassers entstehen. Boussingault hat ferner durch genaue Versuche nachgewiesen, dass gewisse Pflanzen und namentlich gerwisse Leguminosen aus der Atmosphäre einen Theil Stickstoff aufnehmen und assimiliren können.

Die Ernährung der Pflanzen ist sicher einer der interessantesten Punkte ihrer Geschichte. Das Sludium der Untersuchungen, die wir im Vorstehenden in der Kürze angeführt haben, veranlasste uns, einige der angestellten Versuche zu wiederholen. Als wir im Laufe unserer Versuche einige unter dem Wasser lebende Pflanzen in natürlichem Wasser, das Kohlensåure gelöst enthielt, dem Sonnenlichte aussetzten, bemerkten wir nicht ohne Erstaunen, dass diese Palanzen unter dem Einflusse des Sonnenlichtes eine bedeutende Menge Sauerstoff entwickelten. Diese Sauerstoffentwickelung, welche bei den in der Luft lebenden Pflanzen fast unmerklich ist und nur durch die Analyse nachgewiesen werden kann, ist hier sehr deutlich zu bemerken. Die verschiedenen Phasen liessen sich leicht verfolgen, das Auge nahm die geringsten Zufälle wahr und ein Irrthum war nicht 
müglich, da sich bei unscren Versuchen gesunde Pllanzen in den normalen Bedingungen ibrer Existenz befanden. Diese Pflanzen verschafiten uns also eine Gelegenheit, mit der grüssten Wahrscheinlichkeit eines genauen Resultales die merkwürdigen Versuche von Bonnet, Priestley, Ingenhouss, Senebier und Sausure zu wiederholen. IVir stellten diese Versuche im Nuseum d'histoire naturelle in dem Laboratorium Che vreuls und unter dessen Leitung an.

Wie es häufig bei dieser Art von Versuchen vorkommt, aus den zuerst aufgestellten Fragen entstehen neue und der Ilorizont erweitert sich dem Ange des Forsehers immer melre und mehr. Daher ist es denn auch hei unseren Versuchen gekommen, dass dieselben noch keineswegs beendigt sind. Im Laule der Untersuchung haben wir aber einige Beobachtungen gemacht, welche der Beachtung der Physiologen werth zu sein scheinen; wir stehen daher nicht an, tiieselben zu veröffentlichen.

Wenn man einige Blitter einer unter dem Wasser Jebenden Pllanze wie von Potamoyeton oder Naias z. B. in einem langen Probirglase, das mil Kohlensäure gesïtligtes Wasser enthält, umgekchrt in cin Gefäss mit Wasser stellt, und den Apparat dem Sonnenlichte aussetzt, so sieht man augenblicklich von der Oberfläche der Blätter eine grosse Ilenge von kleinen Bläschen sich entwickeln, die aus fast reinem Sautstoffyase bestehen. Dieser Apparat ist so einfach und bequem, dass man vermittelst desselben die Erscheinung der Reduction der Kohlensäure durch die grünen Pflanzentheile leicht zejgen kann; er ist aber nicht genïgend, wenn man nil Genauigkeit die verschiedenen Umstănde, unter denen die Erscheinung vor sich gelit, erschen will.

Der von uns zu den meisten unserer Versuche angewendete Apparat zeigt grosse Vortheile, obgleich er ebenfalls sehr einfach ist. Er bestelit aus einer Flasche von weissem Glase von 4-10 Liter Capacität, das sorgfältig durch einen zweimal durchbohrten Kork, durch welchen zwei Rơhren gehen, verschlossen ist. Die eine dieser Röhren ist gerade und gelt bis auf den Borlen des Gefässes, sie dient zur Ernenerung der Flüssigkeit in dem Innern des Gefässes.

Die zwcite Röhre ist gebogren und wird zum Aufangen des Gases benutzt; ihr in die Flasche reichendes Ende mündet in 
die Spitze eines in die untere Fläche des Korkes eingeschnittenen Hollkegels.

Diese Vorricltung hat zum Zweck, die kleinsten Mengen des ausgegebenen Gases zu bestimmen, das man dadurch austreibr, dass man etwas Wasser in die zweite Rühre giesst.

Nit dem so rorgerichteten Apparat stellten wir einen vorJăufigen Versuch an, dessen Resultate den Beweis liefern künnen, mit welcher Schnelligkeit Wasserphanzen Kohlensiure zu zersetzen im Stande sind.

Den 3. September 1848 bei schünem Weller, als das Thermometer $21^{\circ} \mathrm{im}$ Schatten zeigte, stellten wir mit sechs Stengcln des Potamogeton perfoliatum, die denselhen Ting in der Seine gesammelt und in Wasser aufbewahrt worden waren, Versuche an. Die Totallänge dieser Stengel hetrug 3,45 Veter; sie hatten 143 Bläter und nalunen ein Volunen von 160 Kubjicentimetern ciu.

Diese Stengel wurden in ein Gefïs gebracht, das sechs Liter gewölnliches, mit Kohlensiüre gesilttigtes Gas enthielt. Der Apparat wurde, michslem er wie nben vorgerichtet worden war, dem Sonnenlichte ausgesetzt. Dic Zersetzung fing sogleich an und wir sahen eine unzahllige Henge kleiner Bläschen nach dem oberen Theil des Gefïsses steigen, welche durch das Entwickelungsrohr entwichen. Der Versuch wurle fünf Stunden lang denselben Tag und einen Theil des folgenden Tages fortgesetzt. Das Wasser im Geläss wurde im Anfange des nächstfolgenden Tages erneuert.

Das von den Stengeln ausgebene Gas wurle aufgefangen. In dem Maasse, als die Probirgluser sich anfülten, wurde das in ihnen enthaltene Gas in einc grosse graduirte Glocke geleitct. Nach Beendigung des Versuches, der ungefillr zehn Stunden währte, betrug das Volumen des ausgegebenen Gases bei $15^{0}$ Temperatur und 761 Millimeter Barometerstand 2,35 Liter, was ungelähr das Funfzelnfache vom Volumen der dem Versuche unterworfenen Pflanze ausmacht.

Das bei diesem Versuche erhaltene Gas hatte alle Eigenschafen des Sauerstoffs; es enthielt jeloch eine gewisse Menge Sticksturf beigemengt und ansserdem etwas Kollensaiure. Behufs der Analyse brachten wir ein bestimmtes Volumen in eine Rühre, 
welche eine concentrirte Kalilüsung enthielt. Nachdem die Kohlensäure absorbirt war, wurde der Gasrückstand mit Kupferblech zusammengebracht, das sich in mit einer gleichen Menge Wasser verdünnter Chlorwasserstoffsiare befand. Nach Verlauf einiger Stunden war aller Sanerstoff absorbirt; um aber sicher zu sein, dass die Absorption vollständig vor sich gegangen sei, warteten wir 12 Stunden, ehe wir das Volumen des zurückgebliebenen Gase s bestimmten, das nur aus reinem Stickstoff bestand.

Nach dieser für unseren $Z$ weck hinlänglich genanen Methode, fanden wir, dass 100 Theile des von der Pllanze ausgegebenen Gasgemenges folgende Gase enthielten:

\begin{tabular}{lc} 
Sauerstoff & 87,500 \\
Stickstoff & 11,25 \\
Kohlensiumre & 1.25 \\
\cline { 2 - 2 } & $\frac{100,00}{}$
\end{tabular}

Dieser vorläufige, ơfters und zwar inmer mit ähnlichen Resultaten wiedetholte Versuch enthüllte uns mehrere constante Thatsachen, näınlich: 1) den Einfluss der Intensität des Lichtes; 2) den Einfluss der Temperatur, und 3) die Bildung eines Gasgemenres, dessen Quelle aufgefunden werden musste. Wir wurden 4) veranlasst, Jen Einfluss der umgebenden Mittel auf die Bildung dieses Phänomens zu ermitteln. Aus diesen Thatsachen entstehen einige Fundamentalfratsen, welche wir nach einander zu lösen versuclien wollen.

\section{Einfluss des Lichtes.}

Der Einfluss der lntensitat des Sonnenlichtes auf die Zersctzung der Kohlensiure war schon bei unseren ersten Versuchen deutlich zu bemerken; der Schatten einer kleinen Wolke, die über die Sonne hinwegzieht, ist hinreichend, um die Gasentwickelung zu schwiichen; nachdem die Wolke verschwunden ist, tritt die Entwickelung plützlich wieder mit der früheren Stärke ein. Wir versuchten dieser Beobachtung durch einen Versuch grōssere Schärfe zu gelsen, der darin besteht, die Einwirkung des directen Sonnenlichtes mittelst eines Schirmes za unterbrechen. Die Entwickelung lässt alsbald nach und verschwindet fast gănzlich; dieser Uebergang ist un so melr markirt, je brennender die Sonne und je reiner der Himmel ist. Sobald der Schirm entfernt wird, nimmt die Gasentwickelung jhre frühere Stärke an; die Einwirkung des Sonnenlichtes ist demnach eine fast augenblickliche. 
Die Zersetzung der Kohlensäure durclı die Pflanzen hört im zerstreuten Lichte nicht vollständig auf, sie wird nur schwächer. In der vollkommenen Dunkelheit geht sie nicht vor sich.

Die Resultate, welche wir mit Pllanzen im Dunkeln erhielten, stimmen nicht mit denen der Versuche von Ingenhouss überein. Gervǒhnlich nimmt man an, dass die Pflanzen im Dunkeln Koblensäure ausathmen und dadurch die Luft verunreinigen.

Unsere mit Wasserpflanzen angestellten Versuche führten aber zu einem durchans entgegengesetzten Resultate.

Diese Versuche sind leicht zu wiederholen. Unsere Apparate bestanden in Glasgefässen von 4 Litern Capacität, deren Mündung selır genau geschliffen war, damit ein Glasstōpsel vollkommen schloss. Diese Gefãsse waren mit lufthaltigem destillirten Wasser angefült, enthielten aber keine Kohlensãure. Die zu den Versuchen bestimmten Pflanzen wurden sorgfältig mit Wasser gewaschen und darauf in die Gefisse gebracht; letztere sodann mit dem gefetteten Glasstöpsel verschlossen.

Wir unterwarfen fünf verschiedene Pflanzen zu gleicher Zeit dem Versuche, nämlich: Potamogeton perfoliatum, Potamogeton crispum, Ceratophyllum submersum, Myriophyllum spicatum und endlich Naias maxima.

Nachden die Apparate so vorgerichtet waren, wurden die Glasgefïsse an einen vollkommen finstern Ort gebracht; nach Verlauf von vierundzwanzig Stunden wurde das Wasser in einem jeden der Glasgefāsse vermittelst Barytwasser geprüft und nicht die geringste Spur von Kohlensäure gefunden.

Die Apparate blieben noch acht Tage lang im Dunkeln; der Versuch wurde gegen Ende Juli 1848, bei einer mittleren Temperatur von $17-18^{\circ}$, d. h. bei einer Temperatur, welche für die Vegetation der Pflanzen schr günstig ist, angestellt. Jeden Tag wurde das Wasser in den Gefāsson auf Kohlensäure geprüft, aber nicht die geringste Menge derselben gefunden.

Es war demnach bewiesen, dass die Wasserpflanzen im Dunkeln keine Kohlensāure abgeben. Nimmt man die Genauigkeit der Versuche von Ingenhouss und von Saussure an, die wohl nicht $\mathrm{za}$ bezweifeln ist, so finden wir zwischen den Resultaten dieser Versuche und den unsrigen Widersprūche, welche durch weitere Versuche jedoch zum Verschwinden ge- 
bracht werden künnen. Es ist in der That wahrscheinlich, dass die im Dunkeln von den in der Luft lebenden Pllanzen ausgegehene Kohlensïure, nicht direct durch einen Act entsteht, welcher ler Respiration der Thiere ähnlich ist, durch eine Verlrennung des Kohlenstoffs der I'llanze durch Salierstoff, sondern lass dieselbe vielmelr aus den von der Pllanze absorbirten Flüssigkeiten herrühre; mag mun die Pllanze fortwährend aus dem Boden kohlensäurehaltires Wasser aufnelmen, oder mag sie, nachdem sic aus dem Boten enternt wotlen ist, in ihren Zellon eine grosse Henge mit Kohleusäure gesülligtes Wasser cnthatten, welche sich durch die Absorption eines neurn Gases, wie diese

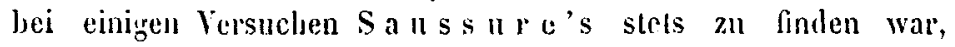
entwickelt.

Im Dunkelı entwickeln temnach die Wasserpflanzen mit lufthalligem Wasser zusammengebracht, licine Liohlensäure. In Sonnenlichte entwickcln dieselben Plianzen eine grosse Menge Saucrstoll, die je nach der Intensitat des Lichtes und der Quantilit der im Wasser gelösten Kollensüure melır oller weniger rein ist.

Nachdem wir nun die Einwirkung des Lichtes in Bezug auf seine Intensität linulänglich geprüt hatten, erschien es uns von Interesse, vergleichungsweise den Einlluss des Lichtes, das durch verschiedengefürbte Gläser gegangen war, zu untersuchen. Zu diesem Zwecke brachten wir die Apparate, welche die Pflanzen enthielten, unter Glaskisten. Der eine dersciben bestand aus ungefürbtem Glase, der zwcite aus mattgeschliffenem Glase, der dritte ans hellgelb gefärbtern Glase, der vierte aus Glas, das durch Kupferoxylul roth gefärbt war, der fünfte aus grünem und ler sechste aus blauem Glase. Die zu unseren Versuchen angewendeten Glasgefässe hatten zwei Jiter Capacität und waren mit gewöhnliclıem Wasser angefüllt, das ungefähr die Hälfte seines Volumens an Kohlensăure enthielt.

Wir brachten in jedes Gefäss dieselbe Anzahl gleichkrältige Blätter, welche in Summe dieselbe Oberfläche darboten. Wir wählten deshalb einen Stengel von Potamogeton perfoliatum, der ungefähr zwanzig Blätter halle, sclnitten den oberen und den unteren Theil der Pllanze ab, an denen die Bläller unglejch gross sind und nahmen nur den mitteren Theil, an welchem die Blätter ziemlich gleiche Oberlläche haben: wir nahmen zwölf 
solcher Theile und zerschnitten sie auf die Weise, dass jedes Blatt an einem Stengelabschnitt von 0,02 Meter Lange sass und brachten in eine jede der sechs Flaschen ein Blatl von jedem Stengel.

Jedes Gefüss enthielt also im Ganzen zwölf blätter, und diese Blätter gehörten zugleich allen angewandten Stengeln an. Diese Vorsichtsmaassregelu waren unerlïsslich, da wir kein anderes ausführbares Mittel kannten, um die Oberfache und die vitale Beschaflenheit der angewandten Pllanzen müglichst gleichmässig zu machen. Jedes Glasgefäss wurde darauf mit eineın mit zwei Röhren versehenen Korke verschlossen; die eine Rühre war so weit, dass ein Weingeist-Thermometer lineingebracht werden konnte; sie ging bis aul den Boden des Gefüsses. Die zweite Röhre war mit Wasser gefüllt und so gebogen, dass das entwickelte Gas unten in ein mit Wasser angefülltes Probirglas geleitet werden konnte. Auf dem Wasser in der zweiten Röhre befand sich eine Schicht Olivenöl.

Die so vorgerichteten Apparate wurden unter die Glaskästen gebracht und dem Sonnenlichte ausigesetat. Nach Verlauf von zwei bis drei Stunden wurde der Versach unterbrochen und die Temperatur, welche das Thermometer in einem jeden liefässe angab, notirt. Darauf wurde das Gas in das Probirglas dadurch geleitet, dass durch die zweite Möhre Wasser in den Apparat gegossen wurde. Das Volumen dieses Gases wurde unter Berücksichtigung der Tenperatur und des Lufturuckes genau bestimmt.

Es blieb uns nur noch die Analyse dieses Gases übrig; zu diesem Zwecke liessen wir die Kohlensäure durch Aetzkali und den Sauerstoff durch Kupferblech bei Gegenwart von verdünnter Chlorwasserstoffsäure absorbiren. 
190 Cloẹz u. Gratiolet: Untersuchungenüb. d. Vegetation

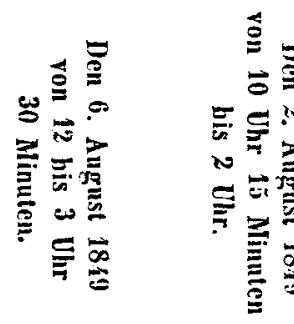

$\stackrel{5}{\Xi}$

今.

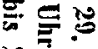

出出

$\cong$

言密

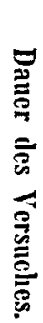

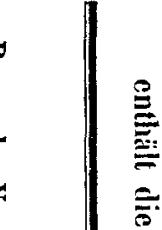

㭉

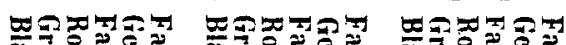

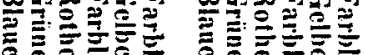

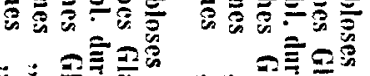

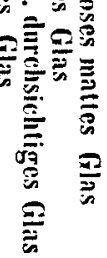

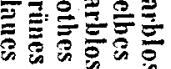

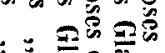

0
0

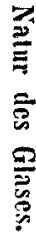

के

.

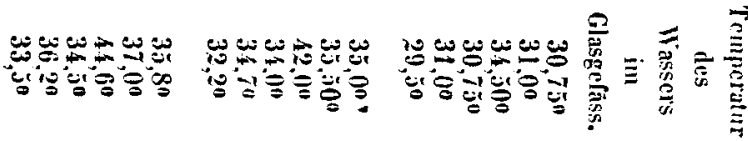

.

$\overline{\mathrm{T}}$

ลั้

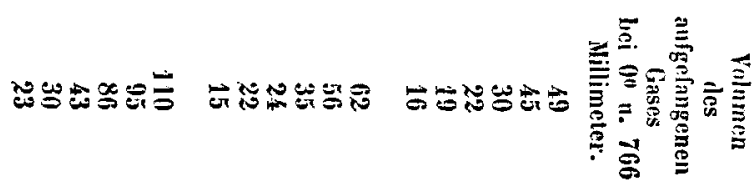

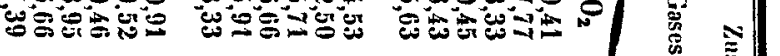

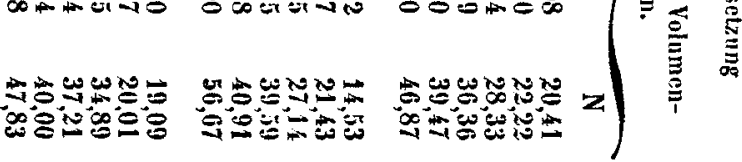

แ

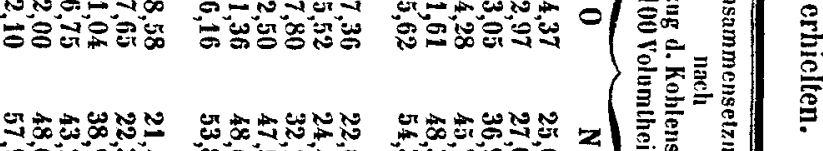

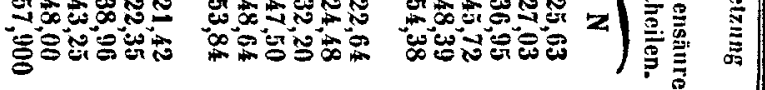


Die beiden letzten Spalten dieser Tabelle geben die Zusammensetzung in 100 Theilen des ausgegebenen Gases nach Abzug der Koblensäure. Wenn man die Zahlen dieser Spalten mit denen vergleicht, welche das Volumen des aufgefangenen Gases ausdrūcken, so findet man, dass das in derselben Zeit und für dieselbe Quantitat der Planze entwickelte Gas von verschiedener Zusammensetzung ist, und dass es um so mehr Sauerstoff enthält, je lebhafter die Reduction der Kohlensäure in den Pllanzen sich zeigt.

Die Zahlen, welche die Volumen des aufgefangenen Gases ausdrücken, geben gewissermaassen den Grad des Einflusses der gefürbten Gläser auf die Lebhaftigkeit des Phänomens an; sie zeigen, dass das Maximum der Einwirkung bei dem mattgeschliffenen ungefärbten Glase statlfindet; nach diesem kommt das gelbe, dann das farblose durchsichtige, das rothe, das grüne Glas, und zuletzt das blauc Glas.

Es köunte nun die Frage entstelsen, ob die Temperatur nicht den wesentlichsten Antheil an den eben angeführten Resultaten gehabt haitte. Vergleicht man dic Zahlen der dritten Spalte mit denen der vierten, so wird man sich überzeugen, dass dem nicht so ist, denn das Gefüss, das sich unter dem farblosen durchsichtigen Glase befindet, war bei unseren Versuchen stets um einige Grad wärmer, als selbst das Thermometer unter dem mattgeschliffenen Glase anzeigte und doch war das Volumen des aufgefangenen Gases unter dem letzteren Glase grōsser als unter dem ersteren.

Man sieht leicht ein, dass, wenn man diese Untersuchungen auf die Luftpflanzen ausdehnt, man aus unseren Versuchen allgemeine Grundsätze für die Construction der Gewächshäuser wird aufstellen können.

Wir künnen uns jedoch nicht verhehlen, dass diese Versuche nur eine Seite der Frage za lösen im Stande sind, nämlich die practische Seite; wir hätten derselben gern eine grössere Schärfe dadurch gegeben, dass wir unsere Pflanzen unter den Strahlen des Sonnenspectrums selbst wachsen liessen; wir mussten aber diese Idee wegen der bedeutenden Kosten, welche die Construction eines passenden Apparates verursacht hătte, aufgeben. 
192 Cloëz u. Gratiolet: Untersuchungen üb.d. Vegetation

\section{Einfluss der Temperatur.}

Bonnot sclirieb die von den Blättern ausgehende Gasentwickelung der durch die Sonnenstrahlen bewirkten Temperaturerhühung zil. Unsere Versuche wie die unserer Vorgänger zeigen, dass eine solche Idee eine irrige ist. Nachdem wir aber den Einfluss des Lichtes auf die in Rede stehende Erscheinung nachgewiesen hatten, blieb uns noch der Einfluss der Temperatur zu ermilteln übrig.

Zu diesem Zwecke brachten wir die Apparate, in welchen sich die Pllanzen in kohlensäurehaitigem Wasser befanden, in ein grosses Glasgefäss, das mit Wasser angefüllt war. In diesem Wasser, zu dem etwas Eis gesetzt wurde, konnten sich die Pflanzen leicht abliühlen. Ein Thermometer ging durch den Kork des Apparates und war dazu bestimmt, die geringsten Variationen der Temperaitur anzugeben.

Durch Modification der Einwirkung des Eises war es uns leicht, abwechseind den Einlluss der gesteigerten Temperatur und den Einhluss des Sinkeus derselben zu ermitteln.

\section{Sleigende Temperalur.}

Unsere Apparate wurden den 27. August 1848 in die Sonne gestellt; das Wasser der Gefïsse war, ehe es dem Lichte ausgesetzt wurde, erst auf clie Temperatur von $+4^{0}$ gebracht worden.

Die Gasentwickelung war anfänglich null. Als die Temperatur des Wassers der $\Lambda$ pparate sich allmählich steigerte, erschienen bei $15^{\circ}$ einige Blasen. Die anfänglich sehr schwache Entwickelung wurde immer stärker, je hüher die Temperatur sich steigerte und schien bei $30^{\circ}$ ilır Maximum erreicht zu haben.

Dieser Versuch wurde mehrere Hale wiederholt und wir erhiplten die Ueberzeugung, dass dic Gasentwickelung nicht unter einer gewissen Temperatur beginnt.

\section{Abnelimende Temperalur.}

Wir brachten das Wasser in den Apparaten auf $30^{\circ}$ und erniedrigten darauf die Temperatur durch Zusatz von Eis. Die Gasentwickelung trat sogleich ein, liess aber allmählich nach. Wir bemerkten jedoch mit Erstaunen, dass die Gasentwickelung bei $15^{0}$ immer noch mit einer gewissen Lebhaftigkeit fortwirkte und erst bei $10^{\circ}$ vollständig beendigt war. 
Diese Versuche lassen sich im Folgenden zusammenfassen. Die Zersetzung der Kohlensãure durch die dem Sonnenlichte ausgesetzten Wasserpflanzen beginnt in einem Mittel, dessen Tcmperatur sich von $+4^{0}$ steigerte, nicht unter $15^{\circ}$ und scheint bei $30^{\circ}$ ihr Maximum zu erreichen; dieselbe Zersetzung geht in einem Mittel, dessen Temperatur von $30^{\circ}$ abnimmt, noch bei $14,13,12$ und $11^{\circ}$ vor sich und hört bei $10^{\circ}$ auf.

Dieses Factum ist für die Physiologie von grosser WVichtigkeit, wenn man es zu den Beobachtungen Cherreul's über die Circulation und das Aulsteigen des Saftes in den Pflanzen in Beziehung bringt. Dieser Chemiker zog aus seinen Versuchen folgenden Schluss: Sobald durch aussere Ursachen die Bewegung des Saftes in den Pflanzen begonnen hat, faliren die Safte fort, ungeachtet einer Abuahme der atmosphärischen Temperatur, sich noch cine Zeit lang zu bewegen, nach Verlauf welcher, wenn die äusseren Umstïnde nicht günstiger für die Vegetation werden, die Bewegung schıächer wird, his sie, nachdem dic ungünstigen Unstande entfernt worden sind, von Neucm beginnt (Journ. des Savants, Mai 1822 p. 302).

Wir künnen hier nicht den Widerspruch mit Stillschweigen übergehen, der zwischen unsereu Beobachtungen und einem von $\mathrm{Liebig}$ in seiner organischen Chenic angegebenen Factum zu bestehen scheint; dieser Chemiker gicbt an, dass während des Winters die grünen Theile der Wasserpflanzen, die sich unter lem Eis befinden, fortwāhrend reines Sauerstoffgas entwickeln, wenn sie von den Sonnenstrahlen getrollen werden. Wir versuchten wăhrend des Winters 1849 mehrere Male diese Reob achtung zil erneuern, doch stets ohne Erfolg. Wir beabsichtigen nicht, die Richtigkeit der von Liebig angegebenen Thatsache in Zweifel zu ziehen, sondern wir glauben, dass über diesen Gegenstand noch Dunkel existirt, das nur durch neue Untersuchungen und neue Beobachtungen zum Verschwinden gebracht werden kann.

\section{Einfluss der Zusammenset:ung des umgebenden Mittels.}

Die Kohlensãure ist augenscheinlich die hauptsāchlichste und unerlässlichste Bedingung für die Erzeugung des Sauerstoffs durch die Pflanzen. Um die Natur des Pliănomens besser studiren zu können, mussten wir dasselbe unter vier verschiedenen Bedingungen 
untersuchen, 1) in natürlichem Flusswasser, in welchem ausser Kohlensãure, noch Gase und Salze gelüst sind; 2) in Flusswasser, aus welchem durch Kochen die Gase ausgetrieben worden sind, das aber noch die Salze enthält und zul welchem man Kohlensäure geselzt hat; 3) in lufthaltigem destillirten Wasser, zu welchem man etwas hohleusäure gesetzt hat; 4) in luftreiem destillirten Wasser, zu welchem etwas liohlensäure gesetzt worden ist und das folglich weder atmosphärische Luft noch Salze enthält.

\section{Resullate der Vegetalion von Wasserplansen in fort- während erneucrten gewölınlichen Wasser.}

Unsere Versuche wurden mit dem schon beschriebenen $A p-$ parate angestellt, durch welchen unaulhurlich ein Strom gewölinJichen Wassers licf. Un Irrthümer zu vermeiden, dic durch die Luftblasen, welche durch den Strom in den Apparat gelïhrt werden, entstehen bümten, brachten wir zwischen dem Apparat und dem Reserroir, in welehem sich das Wasser hefand, eine zweimal tubulirle Flasche an, dis bis anf ein Fünftel mit Wasser angefüllt war. Eine Röhre, die ungeführ 10 Centimeter uuter die Oberfäche des Wassers ging, verband das Glasgetäss mit dem Reservoir; sine zweite Rülure, die bis auf den Boden des Gefässes ging, verband letztcru mit dem Apparat, in welchem sich die Pflanzen befanden. Mit Hälfe dieser Vorrichtung wurden die Gasblasen, welche durch die erste Röhre mit forlgerissen worden waren, in dem oberen Theile des Gefisses zurückgehalten und nur das Wasser konnte durch die zweite Röhre in den übrigen Apparat gelangen.

Nachlem die Pflanzen unter diese Bedingungen gebracht worlen waren, wurde der Versuch sechs Wochen lang mit drei Arten von Pllanzen, nämlich mit Potamogeton perfotiatum, Potamogeton lucens und Naias maxima fortgesetzt. Nach Verlauf dieser Zeit fanden sich die Pflanzen vollikommen gesund und lebenskräftig; sie warcu fast eben so gut gewachsen, als weun sie unter dem Wasser, in welchem sie natürlich leben, durch die Wurzeln am Boden befestigt gewesen wären.

In der ersten Zeit wurde das entwickelte Gas aufgefangen und jeden Tag analysirt; das Volumen und die Zusammensezzung des so erzeuktten Gasgemenges waren je nach der Intensität 
des Lichtes, je nachdem der Himmel rein oder nicht rein und mehr oder weniger nebelig war, für alle dem Versuch unterworfenen Pflanzen veränderlich. Ein constantes Factum liess sich jedoch nachweisen, dass nämlich die Quantităt des in einer gleichen Zeit entwickelten Gases um so grösser und um so reicher an Sauerstoff ist, je lebhafter das Licht war. Es geht diess aus den in folgender Tabelle zusammengestellten Analysen hervor:

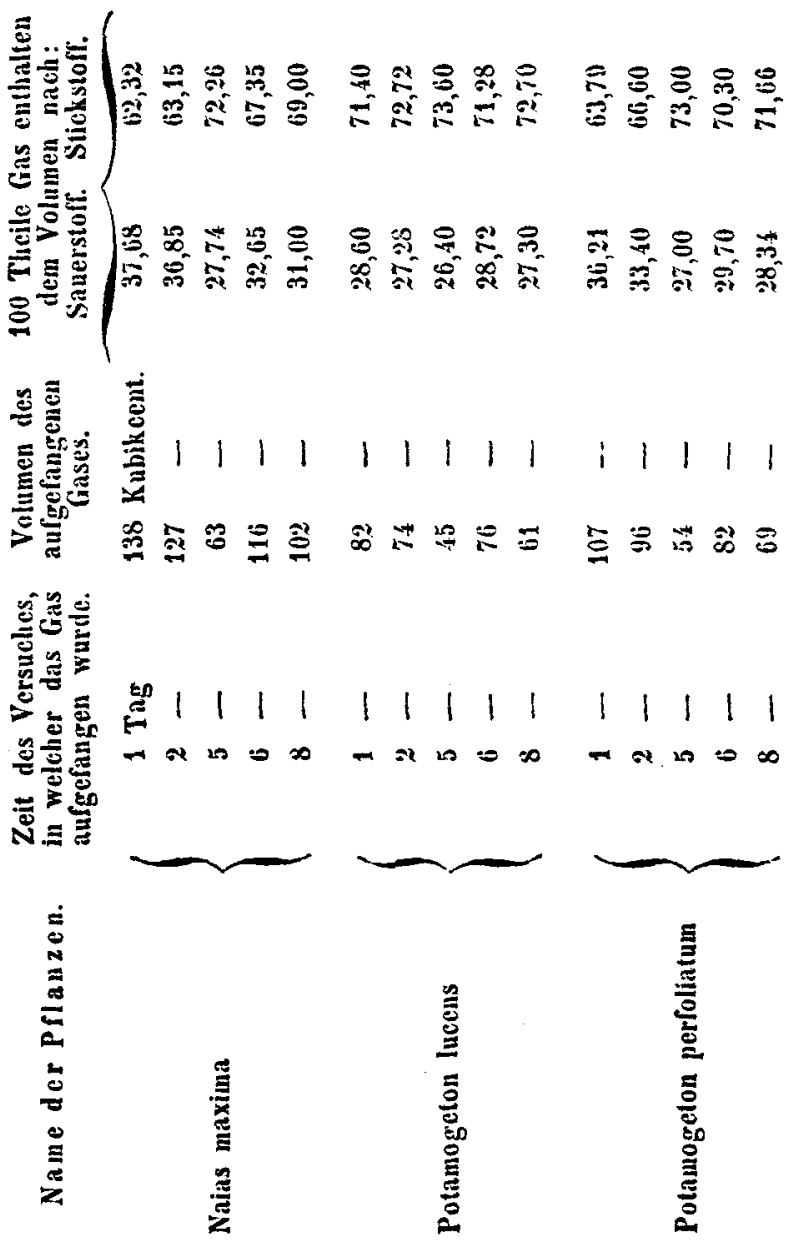


196 Cloéz a. Gratiolet: Untersuchungenüb. d. Vegetation

Diese Tabelle zeigt, dass die relative Menge des durch die Pflanze ausgeschiedenen Stickstoffs sebr gross sein kann; die von uns angestellten Versuche konnten uns aber über die Quelle dieses Gases keinen Aufschluss gelsen. Diese wichtige Frage war der Gegenstand der folgenden Untersuchungen.

Resultate der Vegetation von Wasserpflanzen in natürlichem durch Sieden von der Luft befreilen, aber mit etwas Kohlensäure imprägnirlen Wasser.

Das von der Luft befreite Wasser, das zu unseren Versuchen benutzt wurde, entlielt auf 8 Liter ungeführ 25 Centiliter Kolılensäuregas; das Gals wurde in dem Maase erneuert, als sich Sauerstoff entwickelte, so dass das Wasser ungefähr stets dieselbe Menge Kohlensāure enthielt. $Z u$ diesem $Z$ wecke setzten wir von Zeit zu Zeit eine gewisse Menge von Luft befreiten Wassers hinzu, das eine im Voraus bestimmle Menge Kohlensäure enthielt.

Den 30. Juli 1849 brachten wir in Gefüsse, welche mit diesem so vorbereiteten Wasser angefüllt waren, acht Stengel von Polamogeton perfoliatum, welche zusammen eine länge von 4,80 Neter hatten und 184 Kujikcentimeter Wasser verdrängten.

Den ersten und den zweiten Tag ging die Gasentwickelung, obgleich der Himmel bedeckt war, ziemlich rasch. Den dritten Tag wurde das Welter schün und die Gasentwickelung selrr intensiv; die folgenlen Tage aber liess die Gasentwickelung, obgleich das Wetter gleich schön blieb, nach und wurde endlich den achten Tag unnerklich. Nach Ablauf dieser Zeit hatte die Intensität der grünen Farbe der Blälter bedeutend abgenommen; einige Theile der Blätter schienen selbst vollständig entfärbt zu sein.

Das Volunen des jeden Tag aufrefangenen Gasgemenges ist in der zweiten Spalte der nachstehenden Tabelle angegeben; die dritte und vierte Spalte enthalten die Verhältnisse von Sauerstoff und Stickstoff in 100 Volumentheilen des Gasgemenges. Die letzte Spalte gicbt die Totalmenge des ausgegebenen Stickstoffs an. 
Aufgefangenes Volumen Zusammensetzung Totalvolumen Kubikcentimetern $\quad 100$ Theilen
bei 00 u. $76^{\circ}$ Millim. Sancrstoff Stick

\begin{tabular}{|c|c|c|c|c|c|c|}
\hline & & - son. & & & & \\
\hline Erster & Tag & bei $0^{0}$ u. $76^{\circ}$ Millim. & $\underset{8430}{\text { Sancrstoff }}$ & $\begin{array}{l}\text { Stickstoff } \\
1570\end{array}$ & 546 & bikcen \\
\hline Zweiter & - & 569 & 86.21 & 13,79 & 78.4 & Dinut \\
\hline Dritter & - & 624 & 88,00 & 12,00 & 74.8 & 一 \\
\hline Vierter & - & 315 & 89,74 & 10,26 & 32,3 & - \\
\hline Fünfter & - & 226 & 90,47 & 9,53 & 21,5 & - \\
\hline Sechster & - & 102 & 92,85 & 8,15 & 13,1 & - \\
\hline Siebenter & - & 120 & 95,66 & 4,34 & 5,2 & - \\
\hline Achter & - & 86 & 97,10 & 2,90 & 2,5 & - \\
\hline & & & Tot & nge & 24 & ik \\
\hline
\end{tabular}

Diese Analysen zeizen also 1) dass die relative Menge des in dem ausgegehenen Gasgemenge enthaltenen Sauerstots sich immer melır und mehr vergrŏssert, so Jass gegen das Ende des Versuches das aufgefangene Gas fast reiner Sauerstoff ist; 2) dass das Gesammtrolumen des in den verschiedenen, nach cinander aufgefangenen Gasgemengen weit grösser als das Yolumen der Pllanze ist.

Bei unscren Versuchen konnte dieses Gas nicht von dem Wasser herrülıren, da dasselbe kcinen Stickstoff enthielt; es musste folglich durch die Pflanze selbst erzeugt worden sein; die Pflanze enthielt daher entweder condensirten Stickstoff odler der erzeugte Stickstoff rührte von der Zersetzung der Pflanzensubstanz selbst her.

Um diese beiden Fragen zu lösen, versuchten wir zuerst die Menge des im normalen Zustande in den Pflanzen frei entlaltenen Stickstoffs zu bestimmen. Zu diesem Behufo brachten wir in einen Ballon, der mit Koclısalz gesätligtes Wasser enthielt, acht frische Stengel von Potamogeton, welche zusammen ein Volumen von 196 Kubikcentimetern einnahmen. Mit Hülfe eines Korkes wurde mit dem Ballon eine Entwickelungsrölre verbunden, die glrichfalls mit Salzlösung angefüllt war, und unter eine graduirte Röhre führte, die mit Wasser gefüllt war, auf dessen Oberfläche sich eine kleine Quantität Olivenöl befand.

Das Volumen des durch die Einwirkung der Wärme entwickelten Gases betrug bei $15^{\circ}$ und bei 761 Millimetern 59 Kubikcentimeter. Wir analysirten dieses Gas und fanden es in 100 Theilen zusammengesetzt

$$
\begin{array}{lr}
\text { Kohlensãnre } & \mathbf{4 2 , 5 0} \\
\text { Stickstoff } & \mathbf{5 7 , 5 0} \\
\cline { 2 - 2 } & 100,00
\end{array}
$$


198 Gloèzu. Gratiolet: Untersuchungenüb. d. Vegetation

Diess entspricht ungefähr 33 Kubikcentimetern Stickstoff und ungefähr $1 / 6$ von dem Volumen der Pflanze.

$\mathrm{Da}$ das Yolumen des Stickstoffs weit kleiner als das der Pllanze war, so lässt sich nicht annehmen, dass das Gas in dem Zwischenraume der Stengel die Quelle der grossen, bei den vorstehenden Versuchen erhaltenen Menge Stickstoff sei. Wir mussten dieselle anderswo suchen. Zu diesem Zwecke stellten wir folgenden Versuch an.

Zwanzig Stengel von Potamogeton perfoliatum ron gleicher Leppigkeit und von fast gleicher Grösse, wurden ron denselben Orte in der Seine gesamnelt. Nachdem sie mit destillirten Wasser gewaschen worden waren, wurden sie in zwei Theile getheilt. Zelın Stengel wurden sogleich bei einer Tenperatur von $120^{\circ}$ getroclinet, in Pulver verwandelt und in einem rerschlossenen Gefisse aulhewalirt. Die zelin anderen Stengel wurden in die Apparate gebracht, welche luftfreies und nit Kohlensiuture imprägnirtes Wasser in dem oben angegebenen Verhältnisse enthielten. Nach Verlauf von sechs Tagen liess die Gasentwickelung nach; die Pllauzen wurden aus den Gefässen genommen, mit derselben Vorsicht getrocknet, wie die ersteren ind sodann gepulvert. Als the entwickelte Gas gemessen werden sollte, geschah ein Unlall mit der Glasglocke, so dass ein bedeutender Verlust des Gases stattfand. Glücklicherweise war das Messen das Gases niclit absolut nothwendig.

Um die Menge des in dem Pulver der Pfanze enthaltenen Stickstoffs zu erfalıren, wurde das gewöhnliche Verfahren der Elementaranalyse angewenlet.

Pflanæe, velche unmittelbar nach dem Herausnehmen aus der Seine getrocknet worden war.

0,753 Gr. gepulverte und bei $110^{\circ}$ getrocknete Pllanzen hinterliessen nach dem Eināschern in einer Platinschale 0,115 Gr. Rückstand, entsprechend 15,28 p. C.

1,442 Gr. Jerselben Pflanze gaben bein Verbrennen mit Kupferoxyd 54 Kubikcentimeter Stickstoffgas bei $14^{0}$ Temperatur und 766 Millim. Barometerstand; entsprechend $0,064 \mathrm{Gr}$. oder $\mathbf{5 , 2 3}$ p. C. Stickstofr. 
Pflanze, velche, nachutem sie sechs Tage lang in der Sonne in luftfreiem, mit Kohlensäure leicht imprägnirlen Wasser vegetirt hatte, getrocknet wurde.

1,384 Gr. dieser gepulverten bei $110^{\circ}$ getrockneten Pflanze linterliessen in ciner Platinschale verbrannt $0,183 \mathrm{Gr}$. Rückstand, entsprechend $13,25 \mathrm{p}$. $\mathrm{C}$.

1,202 Gr. derselben Pflanze gaben 39 Kubilicent. Slickgas bei einer Temperatur von $15^{0}$ und 763 Millim. Barometerstand, entsprechend 0,040 $8 \mathrm{Gr}$. Stickstoft oder $3,74 \mathrm{p}$. C.

Diese Analysen zeigen, dass die Pllanze beim Vegetiren in luftreiem Wasser eine beträchtliche Menge Stickstoff auf Kosten ibrer eignen Substanz verlor.

Vergleicht Inan diesen Stickstofrerlust mit der theilweisen Entfäluung am Ende des Versuches, so wird man zu der Annalume veranlasst, dass zwischen dem Aushauchen von Sauerstoff durch die Pllanze und der Zerselzung eines stickstumbltigen Bestanfltheiles, der vielleicht mit der grünen Substanz der Blätler zusammenhängt, eine innige Beziehung stattindet. Wir haben uns vorgenommon, spater in diese Frage tiefer einzugehen, die mit anderen für die Ernälrung der Kräuterfresser so wichtigen Fragen in naher Verbindung steht.

Resullate der Vogelation von Wasserpflan:en in lufthaltigem oder lufifreicm destilliten Wasser, das eine geringe Il'nge Eohlensäure enthielt.

Die Resultate der Versuche in lufthaltigem oder lufffreiem destillirten Wisser, das etwas Kohlensinure enthielt, waren so ziemlich dieselben; es fand jedoch dabci der Unterschied statt, dass die Sanerstofientwickelung in dem Wasser, das Luft gelöst enthielt, länges anhielt. In dem einen wie in dem anderen Falle waren die Pflanzen schnell gebleicht. Es wurde ein Theil der Solze, die in der Pfianze enthalten waren, dadurch ausgeschieden; dieser Theil befand sich im gelösten Zustande in dem umgebenden Wasser. Die dem Versuche unterworfenen, bei $110^{\circ}$ getrockneten und eingeäscherten Pflanzen hinterliessen weniger Asche als dieselben Pllanzen, die nicht dem Versucbe unterworfen worden waren.

Die Resultate der Analyse sind folgende:

$0,910 \mathrm{Gr}$. Polamogeton perfoliatum, welche mit lufthaltigem 
200 Cloëz Gr. Gratiolet: Untersuchungen üb. d. Vegetation

destillirten Wasser behandelt worden waren, linterliessen nach dem Verbrennen in einer Platinschale 0,210 Gr. Rückstand, entsprechend 10,98 p. C.

$1,068 \mathrm{Gr}$. der Pllanze, mil weleher der Versuch in luftreiem destillirten Wasser angestellt worden war, gaben $0,116 \mathrm{Gr}$. Asche, entsprechend 10,86 p. C. Asche.

Endlich 0,950 Cr. der getrockneten Pflanze aus dem Wasser, in welchem sic nalürlich vorkommt unt in derselben Zeit aus dem Wasser entfernt wie dis vorigen, gahen 0,150 Gr. Rückstiml, oder 15,78 p. C.

Fassen wir in ler Kïrze die allgemeinen Resultate aus den in forstehenden beschriebenen firsuchen zusammen, so wird man die Vebergengung gewimnen, dass eines Theils der Stickstoff, anderen Theils die Silze, welche sich in den natïrlichen Wässern gelüst hediulen, eine'n nollwendigen Antheil an der Sauerstoftentwickelung haben, dic ron der Zersitzung der Kohkensäıre durch die grünen Theile der Wasserpflanzen herrührt. Beide bewirken nicht dirert dieses Phänomen, da dasselbe sich anfänglich mit grosser Lebhaftigkeit in einen Wasser, das keine Kohlensäure enthäl, zu crkemnen gieht, sondern indirect auf die Weise, dass sie den Pllanzen gervisse zur Fortsetzung ihres vegetabilischen Lcloens nothwendige Bestandtheile liefern.

Diese Resultate veranlassen forner die Annalume, dass diese Zersetzung unter dem Einflusse gewisser organischer stickstorfhaltiger Verbindungen, die wesentlich veränderlich sind, vor sich gehe. Die Zersetzung der Kohlensätre scheint in einem so holien Grode mit der Zersetzung dieser Substanz verknüpt zu sein, dass die Pfanze, um zu leben, nothwendigerweise die zu ihrer Reproduction noflswendigen Elemente aus den umgebenden Mittelı entnehmen muss. Nach dieser Hypothese, die indess aut positive Beobachtungen sich stützt, sind gewisse Elemente in einem Zustand fortwährender Erneuerung; sie älıneln in dieser Beziehung einigen organischen Elementen der Thiere, die sich ohne Unterlass erneuern.

Es ist hier nicht der Ort, alle Fragen aufzustellen, die sich an dieses Problen knüpfen, das unstreitig eins der wichtigsten der Naturphilosoplie ist.

Wir können jedoch nicht umhin, diesem Stickstoffverlust eine gan $z$ besondere Aultnerksankeit $z \mathfrak{u}$ schenken und werden 
die äussere Ursache dieses für das pflanzliche Leben so wichtigen Elementes zu erfahren suchen.

In den yorstehenden Versuchien, die mit destillirtem, kohlensäurelıaltigen Wasser angestelit worden waren, dauerte die Sauerstoffentwickelung in lufthaltigem Wasser läuger als in luftfreiem; dieser Unterschied konnte nur dem Einfluss des in Wasser gelösten Stickstofls zugeschrieben werden. Anfänglich glaubten wir, dass der freie Stickstoff direct ron der Pflanze assimilirt werden könnte. Es entstand aber nun die Frage, ob diese Quelle dic einzige sei, oder ob der Stickstoff in Verbindung nicht als Nahrungsmittel für die Wasserpflanzen dienen könne. Uin diese Frage aufuklïren, versuchten wir vergleichungstveise die Einwirkung einiger Ammoniaksalze auf Wasserpllanzen, die sich unter den folgenden Bedingungen befanden.

In dem ersten Gefüss befand sich gewöhuliches Jufthaltiges Wasser, welches 25 Centiliter Kohlensäure auf 10 Liter Wasser gelōst enthiclt.

Das zweite Gefïss enthiclt luftreies Wasser, in welchem Kohlensäuregas aufgelöst worden war; es enthielt fernel $1 \mathrm{Gr}$. schwefelsaures Ammoniak auf 10 Liter Wasser.

Das dritte Gefäss enthielt anstalt des schwefelsauren Salzes das salpetersaure Salz derselben Base.

Das vierte endlich enthielt bei gleicher Menge Wasser, die nämliche Quantität koblensaures Ammoniak, d. h. 0,0001 rom Gewichte des Wassers.

Alle Apparate entwickelten anfänglich eine grosse Menge Sauerstoff; die Entwickelung war aber bei Weitem langsamer in den Apparaten, welche Ammoniaksalze enthielten. Nach Verlauf von drei Tagen schritt die Vegetation in den letzten drei Gefässen niclut mehr fort, wälurend sie in dem ersten Apparat noch eben so lebhaft als bein Beginne des Versuches war.

Aus dem ersten Gefäss hatten sich in diesem Zeitraum entwickelt 1765 kubikcent. Gas, aus dem zweiten nur 160 , aus dem dritten 140 und aus dem vierten nur 66 .

Diese Resultate zeigen, dass die Ammoniaksalze, namentlich aber das kohlensaure Ammoniak in der Menge, in der sie angewendet worden sind, einen schädlichen Einfluss ausüben.

Es ist aber möglich, dass diese Dosis zu stark war; wir betrachten deshalb diese Frage nicht als erledigt; die vorge- 
schrittene Jahreszeit gestattete uns nicht, die zur Aultilärung dieses Punctes angestellten Versuche $z u$ beendigen.

\section{Ueber die Bewegung der von den Pfanะen absorbirten und entwickellen Gase.}

Es lässt sich im Voraus schwer angeben, welcher Theil der Blälter die Absorption der Gase und namentlich die der Kohlentsäure bewirkt. Die Structur der beiden Oberflàchen des Blattes zeigt keine bemerkenswerthen Unterschiede; mindestens gelang es uns bis jelzt nicht, dergleichen wahrzunchmen. Eine Beobachtung, welche wir mehrmals gemacht haben, kann aber über dliesen Punct Aulklärung geben.

Wir brachten Stengel von Potamogeton perfoliatum, dessen Blätler auf beiden Seiten vollkommen ruin waren, in eines der. Wasserbassins des Brunnens, desien Wasser eine beträchtliche Menge kohlensauren Kalk in Lüsung enthielt. Diese Vegetabilien fuluren fort zol Jeben, als wenn sie in dem Boden sässen; nach Verlaut aber von ungefïlir vierzehn Tagen bemerkten wil mit Erstaunen, dass alle Blätter, und namentlich die jüngsten, lebenskräftigsten mit einer kreideartigen Kruste überzogen waren. Bei genatterer Prüfung fand sich, dass dieser Absatz sich immer auf der oberen Seite der Blätter beland, während die untere Seite stets vollkommen rein war. Die Idee, dass dieser Absatz nur mechanisch gebildet worken sei, konnte nicht aufkommen, da die Lage der Stengel im Wasser eine fast horizontale war Ind die oberen Flächen der Blätter die entgegengesetzlesten Richtungen hatten. Die verschiedenc Lage, welche sicher auf die Gruppirung der Moleküle, wenn dieselhe durch mechanische Kraft herbeigeführt worden wäre, einen Einlluss geäussert lahben würde, war aber hier, was die Form, das Aussehn und die Dieke des Absatzes anbelangt, ohne Einfluss geblicben. Die Ursache musste also eine andere als eine mechanische sein; um dieselbe aber zu erfahren, war es nothwendig, dass die Natur les Absatzes chemisch ernittelt wurle; eine sehr einfache Analyse zeigte uns, dass diese Substanz nur rciner kohlensaurer Kalk war.

In diesem Factum glauhten wir den Grund der Erscheinung zu finden; es ist bekannt, dass der kohlensaure Kalk durch überschüssige Kollensäure in dem Wasser aufgelöst erhalten wird. Wenn man durch irgend ein Mittel diese überschüssige Kohlen- 
săure abscheidet, so setzt sich der kohlensaure Kalk aus der Lösung $a b$. Dieses Salz verrieth uns also, indem es sich an der oberen Flăche der Blatter absetzt, deutlich, dass hier die Abscheidung, oder mit anderen Worten, die Absorption der Koblensãure durch die Pflanze stattfand.

Nehmen wir das, was wir weiter oben erörtert haben, fiir richtig an, so wird diese Kohlensäure in dem Innern des Blattes durch die Einwirkung des Lichtes auf die grüne Substanz oder einen stickstoffhaltigen Körper, welcher dieselbe begleitet, zersetzt. Wie dem nun auch sei, so steht es fest, dass der abgeschiedene Sauerstoff sich nicht sogleich nach Aussen entwickelt, sondern sich in den Intercellulargängen ansammelt und von da aus zum Theil durch die Seitenporen entwcicht.

Der Lauf dieses Sauerstoffstromes in dem Innern der Pfanze ist ein schr bemerkenswerther; er geht nämlich constant von den Blättern nach den Wurzeln. Es lässt sich diess leicht durch folgenden Versuch nachweisen. Wenn man den mittleren Theil cines Stengels von Poramogeton oder Ceratophyllum in kohlensăurehaltigem Wasser horizontal der Sonne aussetzt, so bemerki man nach einigen Augenblicken aus dem Wurzelabschnitt des Stengels Gasblasen sich entwickeln, während sie an dem anderen Ende unbedeutend und in gewissen Fällen gar nicht zu lemerken ist. Dieser Versuch giebt, man mag den Stengel irgend wclche Lage geben, unverändert stets dassellse Resultat.

Es lässt sich demnach nicht bezweifeln, dass cin Sauerstoflstrom von der Spitze des Stengels nach den Wurzcln strömt. Dieses genat constatirle Phänomen lässt sich auch in seinen Folgen betrachten. Man kann nämlich fragen, ob dieser zu den Wurzeln gehende Sauerstoff keine weitere Rolle bei der Ernährung der Pllanzen spielt, oder ob derselhe vielleicht auf die oxylirbaren organischen Ueberreste, welche die Wurzelu umgeben, einwirkt, und dadurch das Vegetabil fortwährend mit einem Koblensãureruell begabt. Der Ernährungsact der Ptlanze gewăhrt also auch zugleich ein zum Fortbestehen des Lebens nothwendiges Nahrungsmittel.

Schlüsse.

1. Die Zersetzung der Kohlensäure durch die grùnen Theile 
204 Cloëz u. Gratiolet: Untersuchangen ïb.d. Vegetation ete.

der unter dem Wasser lebenden Pflanzen geht nur unter dem Einflusse des Lichtes vor sich.

2. In Dunkeln wird keine Kohlensäure erzeugt.

3. Das durch gefärbte Gläser veründerte Licht hat eine Intensitât, die in folgender Ordnung abnimmt:
a) mattes gescbliffenes farbloses Ghas;
b) gelbes Glas;
c) farbloses durchsichtiges Glas;
d) rothes Glas;
e) grünes Glas;
f) blaues Glas.

4. Zur Entstehung dieses Phänomens ist eine gewisse Temperatur erforderlich; bei steigenter Temperatur beginnt es nicht unter $15^{0}$; bei abnehmender setzt es sich bis unter diese Temperatur his $10^{\circ}$ ither Null fort.

5. Die Salze und die Luft, welche sich nebst der Koblensïure in den natürlichen Wässern finden, sind zur dauernden Eischeinung unerläslich.

6. Das durch dic Pflanzen entwickelte Gas enthält ausser Sauerstoff eine gewisse Mlenge Stickstoff. Dieser Stickstoff rührt zum grüssten Theile von der Zersetzung der Pllanzensubstanz selbst her.

7. Der Stickstoff der Luft, welchen das Wasser gelöst enthält, schcint zur Ausgleichung dieses Verlustes bestimmt zu sein; seite Gegenwart ist unbedingt nothwendig.

8. Das Ammoniak und die Ammoniaksalze, welche sich im Wasser in der Menge von $\mathbf{0 , 0 0 0 1}$ gelöst befinden, bewirken selıt bald den Tod der Pflanzen.

9. Die Ahsorption der Kohlensäure geht nur auf der oberen Fläche der Blätler vor sich.

10. Der Sauerstoff, der von der Zersetzung der Koblensãure herrührt, geht in die Intercellulargefässe und richtet seinen Lauf beständig vou den Blättern nach den Wurzeln. 